\title{
Altered Specificity in DNA Binding by the lac Repressor: A Mutant lac Headpiece that Mimics the gal Repressor
}

\author{
Roberto Kopke Salinas, ${ }^{*[a, b]}$ Gert E. Folkers, ${ }^{[a]}$ Alexandre M. J. J. Bonvin, ${ }^{[a]}$ \\ Devashish Das, ${ }^{[a]}$ Rolf Boelens, ${ }^{[a]}$ and Robert Kaptein ${ }^{*[a]}$
}

\begin{abstract}
Recognition of the lac operator by the lac repressor involves specific interactions between residues in the repressor's recognition helix and bases in the DNA major groove. Tyr17 and Gln18, at positions 1 and 2 in the lac repressor recognition helix, can be exchanged for other amino acids to generate mutant repressors that display altered specificity. We have solved the solution structure of a protein-DNA complex of an altered-specificity mutant lac headpiece in which Tyr17 and Gln18 were exchanged for valine and alanine, respectively, as found in the recognition helix of the gal repressor. As previously described by Lehming et al.
\end{abstract}

(EMBO J. 1987, 6, 3145-3153), this altered-specificity mutant of the lac repressor recognizes a variant lac operator that is similar to the gal operator Oe. The mutant lac headpiece showed the predicted specificity and is also able to mimic the gal repressor by recognizing and bending the natural gal operator Oe. These structural data show that, while most of the anchoring points that help the lac headpiece to assemble on the lac operator were preserved, a different network of protein-DNA interactions connecting Ala17 and Val18 to bases in the DNA major groove drives the specificity towards the altered operator.

\section{Introduction}

Protein-DNA complexes play essential roles in a large range of cellular processes such as translation, regulation of gene expression, DNA repair, and replication. Understanding the mechanism of these processes requires insight into how proteins recognize DNA and form specific or nonspecific complexes. The lac repressor plays the central role in the regulation of gene expression in the lac operon of E. coli, the classical example of transcription regulation in prokaryotes. The large amount of structural and biological data available ${ }^{[1]}$ make the lac repressor an ideal model system with which to study the molecular basis of protein-DNA recognition.

The lac repressor consists of four subunits of 360 amino acids each; DNA-binding surfaces are formed by two dimeric units. The DNA-binding domain, known as the lac headpiece, is located at the $\mathrm{N}$ terminus of the protein (residues 1-62). It belongs to the family of helix-turn-helix (HTH) proteins that are common among transcription regulators. ${ }^{[2]}$ The second helix of the HTH motif is known as the recognition helix. Specific interactions between amino acids in the recognition helix and the major groove of the DNA are a typical feature of protein-DNA recognition for several bacterial and phage transcription regulators. ${ }^{[2-3]}$

NMR and mutagenesis studies have shown that the lac headpiece recognizes the lac operator through a network of interactions that occur between the recognition helix of the repressor and the DNA in the major groove. ${ }^{[4]}$ DNA binding is concomitant with folding of the $\mathrm{C}$-terminal segment (residues $52-58$ ) of the lac headpiece into an $\alpha$-helix that penetrates into the minor groove and causes bending of the DNA. ${ }^{[5]}$ Other important sites of protein-DNA recognition are located in the loop connecting the recognition helix to the third helix and at the N-terminal part of the first helix. However, structural and biological data showed that most of the specific contacts between the lac repressor and the major groove of the lac operator are made by the side chains of Tyr17, Gln18, and Arg22 at positions 1, 2, and 6 of the lac repressor recognition helix, respectively. ${ }^{[4 b-d, g]}$

Addressing the specificity of the lac repressor, Müller-Hill and co-workers performed an in vivo screen, thereby identifying several complementary mutations both on the repressor and on the lac operator, and tested their ability to repress the expression of the lac $Z$ gene coding for $\beta$-galactosidase. ${ }^{[4 c-e, 6]}$ These studies found evidence that several mutant lac repressors with substitutions at positions 1 and 2 of the recognition helix are able to recognize lac operator variants with substitutions in the base pair at position 7, while mutant repressors with substitutions at position 6 of the recognition helix recog-

[a] R. Kopke Salinas, Dr. G. E. Folkers, Dr. A. M. J. J. Bonvin, D. Das, Prof. Dr. R. Boelens, Prof. Dr. R. Kaptein

Department of NMR Spectroscopy

Bijvoet Center for Biomolecular Research, Utrecht University

Padualaan 8, 3584CH Utrecht (The Netherlands)

Fax: $(+31) 30-253-7623$

E-mail:kaptein@nmr.chem.uu.nl

[b] R. Kopke Salinas

Current address:

Department of Biochemistry

Institute of Chemistry, University of São Paulo

Postal Box 26077, 05513-970 São Paulo SP (Brazil)

Fax: (+55) 11-3815-5579

E-mail:roberto@iq.usp.br

Supporting information for this article is available on the WWW under http://www.chembiochem.org or from the author. 
A)

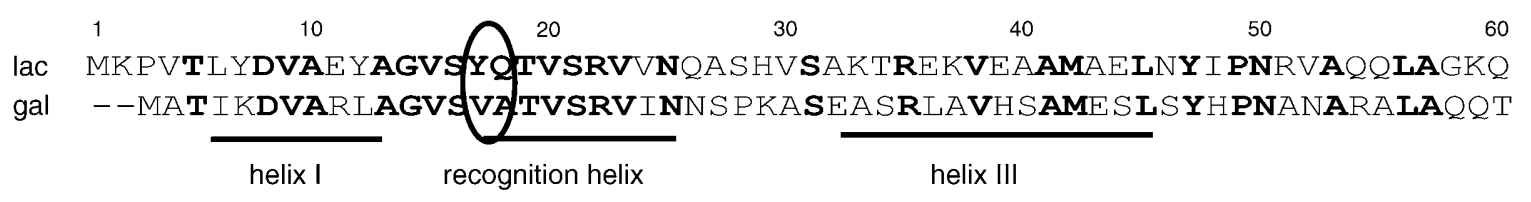

B)

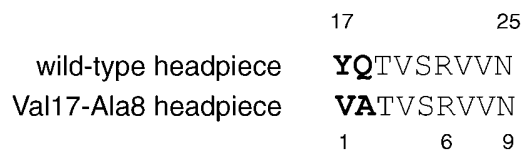

C)

$$
\begin{aligned}
& \text { lac-gal } \\
& \text { lac-SymL }
\end{aligned}
$$$$
\text { NOD }
$$

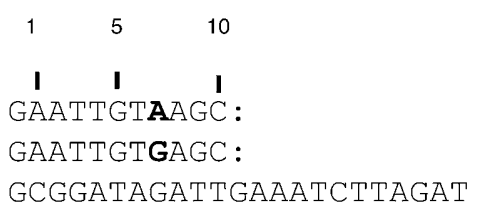

D)

lac-gal

gal Oe

$\begin{array}{ccccc}1 & 5 & 10 & 16 & 20 \\ \text { I } & \text { I } & \text { I } & \mathbf{I} & \mathbf{I} \\ \text { GAATTGTAGC } & \text { GCTTACAATTC } \\ \text { CTTGTAAAC } & \text { GATTCCACTAA }\end{array}$

Figure 1. A) Sequence alignment of the DNA-binding domains of lac (top) and gal (bottom) repressors. The residues at positions 17 and 18 of the recognition helices (numbering of the lac repressor) are circled, conserved residues are shown in bold. B) Comparison of the amino acid sequences of the recognition helices (residues 17-25) of the wild-type lac headpiece and the Val17Ala18 headpiece. The numbering scheme of the lac repressor is shown at the top, and Müller-Hill's numbering scheme at the bottom. The residues 17 and 18 (or 1 and 2 according to Müller-Hill's numbering scheme ${ }^{[4]}$ ) are shown in bold. C) Nucleotide sequences of the lac-gal, the lac-SymL, and the NOD operators. The different base pairs at position 7 of the lac-gal and SymL operators are depicted in bold. All the operators are perfect palindromes and contain two identical binding sites for the protein, so only one half site is shown and the center of symmetry is symbolized by a double dot. Lac-gal is the operator specific for the Val17Ala18 headpiece, while lac-SymL, also called ideal lac operator, is a palindrome of the left side of the natural lac operator $\mathrm{O} 1$ with the central base pair deleted. NOD is a nonspecific operator. D) Comparison of the nucleotide sequences of the lac-gal and gal Oe operators. The identical base pairs are shown in bold. The center of symmetry is symbolized by a double dot.

nize variant lac operators with different base pairs at position 5 of the lac operator. Some of these mutants bind better to the variant operator than to the ideal lac operator, ${ }^{[7]}$ which indicates a change of DNA specificity. This was the case for mutant lac repressors in which Tyr17 and Gln18 at positions 1 and 2 of the recognition helix had been exchanged for those amino acids found in the recognition helix of the gal and the deo repressors. These lac repressor mutants showed specificity for variant lac operators in which the GC7 base pair had been altered for the base pair found in the equivalent position of the gal and deo operator, respectively. ${ }^{[4,8]}$

In order to understand the altered specificity of the mutant lac repressor, we constructed a mutant lac headpiece in which the residues at positions 1 and 2 of the recognition helix were exchanged for valine and alanine found in the equivalent positions of the gal repressor (Figure 1 A, B). Lehming et al. (1987) demonstrated that in vivo the Val17Ala18 lac repressor would recognize a variant of the ideal lac operator containing an AT base pair at position 7 (here called lac-gal operator; Figure $1 \mathrm{C}$, D) much better than the wild-type lac repressor. ${ }^{[4 c]}$

In this paper, we describe the NMR structure of a proteinDNA complex formed between the Val17Ala18 lac headpiece and the lac-gal operator. The structure shows that, while the anchoring contacts between the lac headpiece and the lac operator are preserved, the specificity is determined by a new network of protein-DNA interactions in the major groove of the DNA. The results are discussed in relation to the wild-type lac system and provide further information for understanding the role of the residues at positions 1 and 2 of the lac repressor recognition helix in the determination of DNA specificity.

\section{Results}

\section{Relative in vitro affinities and DNA bending}

A mutant lac headpiece in which Tyr17 and Gln18 at positions 1 and 2 of the recognition helix had been exchanged for Val and Ala, respectively, as found in the recognition helix of the gal repressor (Figure 1A, B), was overexpressed in $E$ coli. The mutant also contains the substitution V52C, which allows the protein to be isolated as a covalent dimer under oxidizing conditions. As previously described, the dimeric lac headpiece binds much more tightly than the monomeric form to the lac operator. ${ }^{[9]}$

From the work of Lehming et al. (1987), the Val17Ala18 lac headpiece should specifically recognize the lac-gal operator, which differs from the ideal lac operator at position 7, and further differs from the left side of the gal Oe operator at positions 9 and 3 (Figure 1C, D). ${ }^{[7,8 c, 4 c]}$

The DNA specificity of the Val17Ala18 headpiece was studied by gel retardation assays. The experiments performed with both the lac-gal operator and the ideal lac operator (lac-SymL 


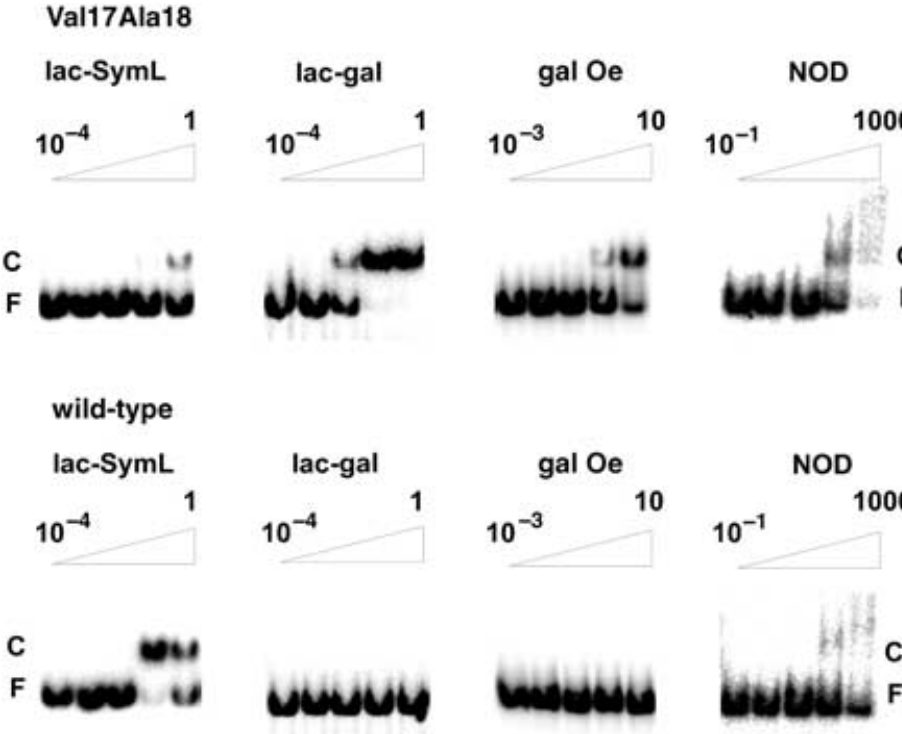

Figure 2. Representative DNA-binding experiment with the indicated operators, with serial dilution of the dimeric Val17Ala18 mutant (upper part) or wild-type (lower part) lac headpiece under high salt conditions $(250 \mathrm{~mm}$ $\mathrm{KCl})$. The highest and lowest protein concentrations [nM] are indicated above each experiment. The different operator fragments lac-gal, lac-SymL, gal Oe, and NOD are described in Figure $1 \mathrm{C}$. The free probe and the protein-DNA complex are indicated by $F$ and $C$, respectively

operator) showed that the Val17Ala18 headpiece binds to the lac-gal operator, whilst the wild-type headpiece does not (Figure 2). Binding experiments showed that the apparent equilibrium dissociation constants $\left(K_{\mathrm{d}}\right)$ of both the wild-type headpiece and the Val17Ala18 headpiece for the lac-SymL and the lac-gal operators, respectively, are in the $\mathrm{nm}$ range (Figure 2, Table 1). The high affinity of the Val17Ala18 lac headpiece for the lac-gal operator observed here provides an explanation for the 200-fold increase in repression of $\beta$-galactosidase activity observed in the presence of the Val17Ala18 lac repressor in relation to the wild type, when the transcription of the lacZ gene was under control of the lac-gal operator. ${ }^{[4 c]}$

Remarkably, control experiments showed that the Val17Ala18 headpiece also binds to the lac-SymL operator under low salt conditions. However, at high salt concentrations $(250 \mathrm{~mm} \mathrm{KCl})$ the binding is strongly reduced (Figure 2, Table 1).

Furthermore, we observed that the Val17Ala18 headpiece recognizes the natural gal Oe operator while the wild-type lac headpiece does not (Figure 2). However, binding to the gal Oe operator is also strongly reduced by increasing salt concentration. In all cases the apparent $K_{d}$ values were at least one order of magnitude smaller than those observed for binding to a nonoperator DNA (NOD; Figure 1C, Table 1).

Protein-induced DNA bending was investigated by using the circular permutation method of $\mathrm{Wu}$ and Crothers on complexes with the lac-gal, the lac-SymL, and the gal Oe operators. ${ }^{[10]}$ The profile of the migra-

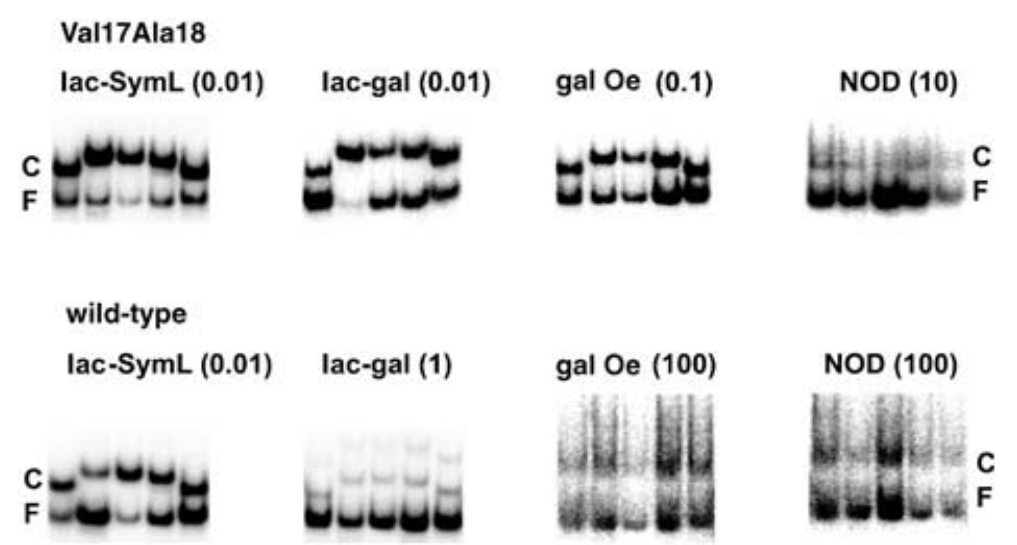

Figure 3. Representative circular-permutation-method bending experiments with the dimeric Val17Ala18 mutant (upper part) or the wild-type (lower part) lac headpiece under standard conditions $(50 \mathrm{~mm} \mathrm{KCl})$, through the use of the indicated amount of protein [nм] with the operator fragments lac-gal, lac-SymL, gal Oe, and NOD as described in Figure $1 \mathrm{C}$. The free probe and the protein DNA complex are indicated by $\mathrm{F}$ and $\mathrm{C}$, respectively. 
Interestingly, the Val17Ala18 headpiece is also able to bend the gal Oe operator by approximately $35^{\circ}$ upon binding (Figure 3), mimicking the behavior of the gal repressor, ${ }^{[13]}$ while the wild-type lac headpiece fails to induce a bend in this operator.

\section{Structure description}

The tight binding between the dimeric Val17Ala18 headpiece and the lac-gal operator resulted in a complex in slow exchange on the NMR timescale. Because of the C2 symmetry of the complex, only one set of 59 cross-peaks corresponding to backbone amide atoms was observed in the ${ }^{1} \mathrm{H},{ }^{15} \mathrm{~N}$ HSQC spectrum. The solution structure of the complex was solved by heteronuclear double- and triple-resonance NMR techniques, as described in the Experimental Section. The ensemble containing the 16 lowest-energy structures is shown in Figure 4. The statistics for the structures are described in Table 2.
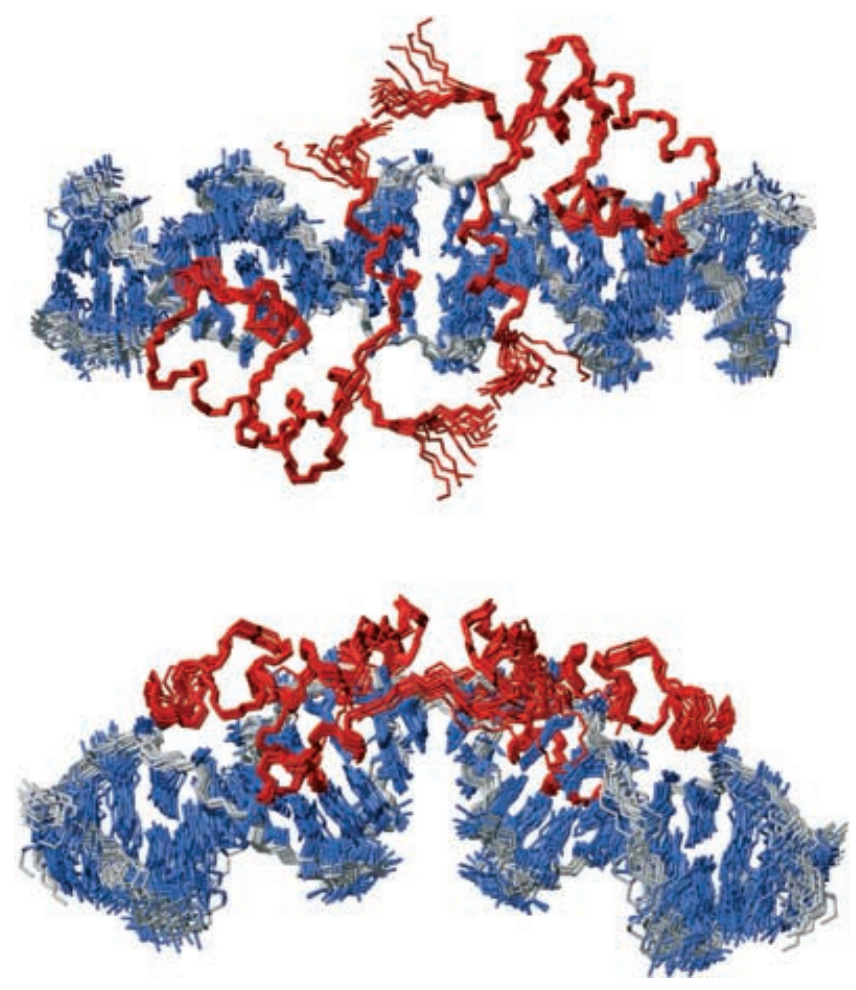

Figure 4. Ensemble of the 16 lowest-energy structures of the Val17Ala18 headpiece-DNA complex shown in two different views. Backbone atoms from amino acids $4-58$ of the two monomers and base pairs 2-21 of the DNA were used for the superposition. The protein is shown in red, and DNA bases in blue. The DNA backbone is shown in gray.

The structure of the mutant lac headpiece bound to DNA contains four $\alpha$-helices: residues $5-13,17-25,32-45$, and $53-$ 58. An overlay with the structure of the free lac headpiece shows that the root mean squared deviation (RMSD) of the positions of the backbone atoms $\left(\mathrm{C}_{\alpha}, \mathrm{N}\right.$, and $\left.\mathrm{C}^{\prime}\right)$ of amino acids 525 and $32-45$ is $0.90 \pm 0.01 \AA$, indicating that the structure of the three-helical globular subdomain of the Val17Ala18 head-
Table 2. Structural statistics.

R.m.s.deviation $[\AA]$ of the backbone/all heavy atom coordinates. ${ }^{[a]}$

\begin{tabular}{ll}
\hline Val17Ala18 headpiece-DNA complex & $0.54 \pm 0.15 / 0.74 \pm 0.18$ \\
Protein & $0.38 \pm 0.15 / 0.65 \pm 0.14$ \\
DNA & $0.80 \pm 0.25 / 0.73 \pm 0.24$
\end{tabular}

Number of experimental restraints (for each protein monomer and DNA half site)

Distance restraints

intraresidue NOEs

medium-range NOEs $(2<|i-j|<4) \quad 251$

long-range NOEs $(|i-j|>5)$

intermonomer NOEs $\quad 19$

DNA NOEs 294

intermolecular NOEs

intermolecular $\mathrm{H}$ bonds

dihedral angle restraint ${ }^{[\mathrm{b}]}$

dipolar coupling restraints $\left(D_{H N}\right) \quad 49$

R.m.s. deviation from experimental restraints

all distance restraints $[\AA]$ : $\quad 0.053 \pm 0.001$

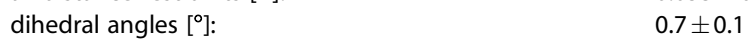

$\begin{array}{ll}D_{\mathrm{HN}}[\mathrm{Hz}] & 0.076 \pm 0.003\end{array}$

Average $\mathrm{Q}$ factor for dipolar coupling restraints ${ }^{[\mathrm{c}]}$

$\begin{array}{ll}D_{\mathrm{HN}} & 0.09 \pm 0.01\end{array}$

$\begin{array}{ll}D_{\mathrm{HN}}[\mathrm{d}] & 0.09 \pm 0.01 \\ D_{\mathrm{C \alpha} \mathrm{H \alpha}} & 0.49 \pm 0.02\end{array}$

Experimental restraints

violations

distances with violations $>0.3 \AA \quad 2.8 \pm 2.0$

$\begin{array}{ll}\text { dihedral angles with violations }>5^{\circ} & 0.4 \pm 0.7\end{array}$

$\begin{array}{ll}D_{\mathrm{HN}} \text { with violations }>0.2 \mathrm{~Hz} & 3.2 \pm 0.8\end{array}$

R.m.s. deviation from idealized geometry

bonds $[\AA]$

$\begin{array}{ll}\text { impropers }\left[{ }^{\circ}\right] & 0.67 \pm 0.02\end{array}$

Ramachandran analysis [\%]

residues in the most favored regions $\quad 83.8 \pm 2.2$

residues in additional allowed regions $\quad 9.9 \pm 2.6$

residues in generously allowed regions $\quad 4.2 \pm 1.4$

$\begin{array}{lr}\text { residues in disallowed regions } & 2.2 \pm 1.2\end{array}$

CNS energies ${ }^{[\mathrm{e}]}$

Lennard-Jones $\left[\mathrm{kcal} \mathrm{mol}^{-1}\right] \quad-742 \pm 29$

electrostatic $\left[\mathrm{kcal} \mathrm{mol}^{-1}\right] \quad-5620 \pm 131$

[a] RMSD values were calculated by use of PROFIT (http://acrmwww. biochem.ucl.ac.uk/software/profit), fitting on the backbone included the $\mathrm{N}, \mathrm{C}_{\alpha}$ and carbonyl atoms of residues 4-58 from the two monomers and $\mathrm{C}^{\prime}$ and $\mathrm{P}$ atoms of the base pairs 2-21 from the DNA. [b] Dihedral angle restraints were derived from TALOS predictions of $\varphi$ and $\phi$ angles (see Experimental Section). ${ }^{[28]}$ [c] The Q-factors were calculated with PALES. ${ }^{[30 c]}$ [d] The $D_{\mathrm{CaH} \alpha}$ dipolar couplings were not included in the calculations. [e] The nonbonded energies were calculated by use of the OPLS nonbonded parameter set with a cutoff of $8.5 \AA$.

piece is not significantly different from that of the lac headpiece in the free form. ${ }^{[14]}$

The overall fold, the positions, and the relative orientations of the $\alpha$-helices in the complex are the same as in the wild- 
type headpiece bound to the lac operator. ${ }^{[49,11,15]}$ Comparison with the structure of the wild-type lac headpiece bound to the left side of the lac operator ${ }^{[4 g]}$ gives a backbone RMSD $\left(C_{\alpha}, N\right.$, and $C^{\prime}$ ) of $0.97 \pm 0.06 \AA$ for amino acids $5-58$, while the RMSD of the backbone atoms in the HTH domain $\left(C_{a}, N\right.$, and $C^{\prime}$ of amino acids $5-25)$ is $0.43 \pm 0.03 \AA$. The recognition helix binds the major groove, while the hinge helix interacts in the minor groove forming the interface between the two monomers. These orientations are compatible with the measured $\mathrm{HN}$ residual dipolar couplings (RDCs) based on the intrinsic anisotropic magnetic susceptibility of the DNA at high magnetic fields (see Supporting Information).

The starting structure for the lac-gal operator was a regular DNA in the B-form. However, docking of the protein onto the DNA resulted in a large kink in the DNA structure (Figure 4). Analysis of the DNA helical parameters shows large deformations around the central base pair (see Supporting Information). The central base pair step $\mathrm{C}^{10} \mathrm{pG}^{11}$ displays an unusual roll angle of $56^{\circ}$, a helical rise of $5.3 \AA$, and a small twist of $12^{\circ}$; this indicates that the DNA is unwound around the central base pair. The average bend angle of the DNA in the structures in the ensemble is $42 \pm 11^{\circ}$ as estimated with CURVES. ${ }^{[16]}$ As observed in the wild-type complex, the deformation of the DNA is the result of the double insertion of the side chain of Leu56 between the central base pair step $\mathrm{C}^{10} \mathrm{pG}^{11}$.

Experimentally, the degree of DNA bending can only be assessed by the use of long-range structural information such as RDCs. Although we do not have RDCs for the DNA, the RDCs measured on the protein could also contain information about the DNA bending. An estimate of the alignment tensor parameters resulted in a rhombicity of $0.6 \pm 0.1$; the diagonal alignment tensor components are $A_{x x}=-(0.03 \pm 0.01) \times 10^{-4}, A_{y y}=$ $-(1.00 \pm 0.01) \times 10^{-4}$, and $A_{z z}=(1.04 \pm 0.01) \times 10^{-4}$. Prediction of the axial and rhombic components of the protein-DNA complex magnetic susceptibility tensor based on our structure yields a rhombicity of approximately $0.45 \pm 0.06$ (see Supporting Information). This value by itself indicates a larger degree of asymmetry than would be expected for an axially symmetric DNA. ${ }^{[17]}$

The quality factor (Q-factor), ${ }^{[18]}$ given by fitting of the $D_{\mathrm{CaHo}}$ data (which were not included in the structure calculations), for the structures is $0.49 \pm 0.02$ (Table 2). Taking into account that, due to the larger experimental errors on the measurement of small ${ }^{1} J_{\mathrm{C} \alpha \mathrm{H \alpha}}$ scalar couplings, a Q-factor as large as 0.3 for the $D_{\mathrm{Ca} \mathrm{H \alpha}}$ data was obtained when those data were included in the calculations, the value of 0.49 indicates a reasonable agreement between the structures and the experimental data.

\section{Protein-DNA interactions}

An analysis of the intermolecular interactions found in the ensemble shows how the Val17Ala18 headpiece recognizes the operator. Extensive protein-DNA contacts are made by the residues from the recognition helix and the loop that connects the third helix to the major groove, while the residues of the hinge helix make extensive contacts with the bases in the minor groove (Figure 5).
The positioning of the recognition helix with its $\mathrm{N}$-terminal part perpendicular to the major groove exposes the side chains of Val17 and Ala18, at positions 1 and 2, to a hydrophobic pocket formed by the methyl groups of Thy6, Thy7, and Thy8 (Figure 5).

Ala18 is closest to the altered base pair AT7 (Figure 5). Ala18 $\mathrm{H}_{\alpha}$ is in close contact with the methyl group of Thy7. The $\beta$ $\mathrm{CH}_{3}$ group, which is within van der Waals distance of the amide protons of Ade6 (Figure 5), establishes hydrophobic interactions with the methyl group of Thy6, and is also in close proximity to the amide group of Gua6.

Furthermore, the side chain of Val17 makes intra- and intermolecular contacts. One of the methyl groups of Val17 stacks with the aromatic ring of Tyr7 in all the structures. The other $\mathrm{CH}_{3}$ group, which is located at van der Waals distance of the amide groups of Ade7 (Figure 5E, F), makes hydrophobic interactions with the methyl groups of Thy 6 and Thy8. Val17 also contacts the Thy 8 methyl group with its carbonyl oxygen.

The position of Tyr7 is well defined by ten intermolecular NOEs to the DNA. The orientation of the Tyr7 aromatic ring is not altered by the interaction with the $\gamma 1-\mathrm{CH}_{3}$ group of Val17. The orientation observed in the wild-type complex is conserved, and Tyr7 makes specific contacts to Cyt9 through a hydrogen bond to its amino group (Figure 5).

The altered base pair AT7 also interacts with Ser21. The Ser21 hydroxyl group makes a hydrogen bond to the phosphate of Thy8, and the Ser21 $\beta-\mathrm{CH}_{2}$ group enters into hydrophobic interactions with the methyl groups of Thy 8 and Thy 7 (Figure 5).

As can be observed in Figure 5, most of the remaining protein-DNA contacts made by the other residues of the first helix, the recognition helix, and the loop connecting the recognition helix to the third helix are also observed in the structure of the wild-type complex. The orientations of these side chains-Thr5, Leu6, Ser16, Thr19, Asn25, His29, and Thr34-are therefore conserved in both structures. Moreover, the detailed conformation of the side chains of Tyr47, Asn25, His29, Ser16, and Thr19 is very similar to the conformation observed in the wild-type complex; the conformations of Leu6 and Thr5, however, are slightly different.

The NMR structure of the lac headpiece bound to the natural operator $\mathrm{O} 1$ shows that Arg22 amino groups form hydrogen bonds to $\mathrm{O} 6$ of Gua6 on both the left- (Figure 5) and the right-hand sides, ${ }^{[4 b, g]}$ and this orientation is in agreement with biological experiments that showed this side chain to be essential for specific recognition of the lac operator. ${ }^{[4 d-e]}$ Here the side chain of Arg22 is found to be very dynamic, and only part of the time does it form a $\mathrm{H}$-bond to Gua5 $\mathrm{O6}$, a phenomenon also observed in previous complexes.

From the hinge helix, Ala53, Leu56, and Ala57 are engaged in extensive hydrophobic interactions with the DNA, contacting both the bases and the sugars of Cyt9, Gua10, and Gua10' on the right side of the lac-gal operator. Asn50 and GIn54 form hydrogen bonds with the DNA backbone, while the Ala53 carbonyl group forms a hydrogen bond with the amide group of Gua10. The mechanism of DNA bending is therefore the same as in the lac operator. Insertion of the hinge helix 
A)
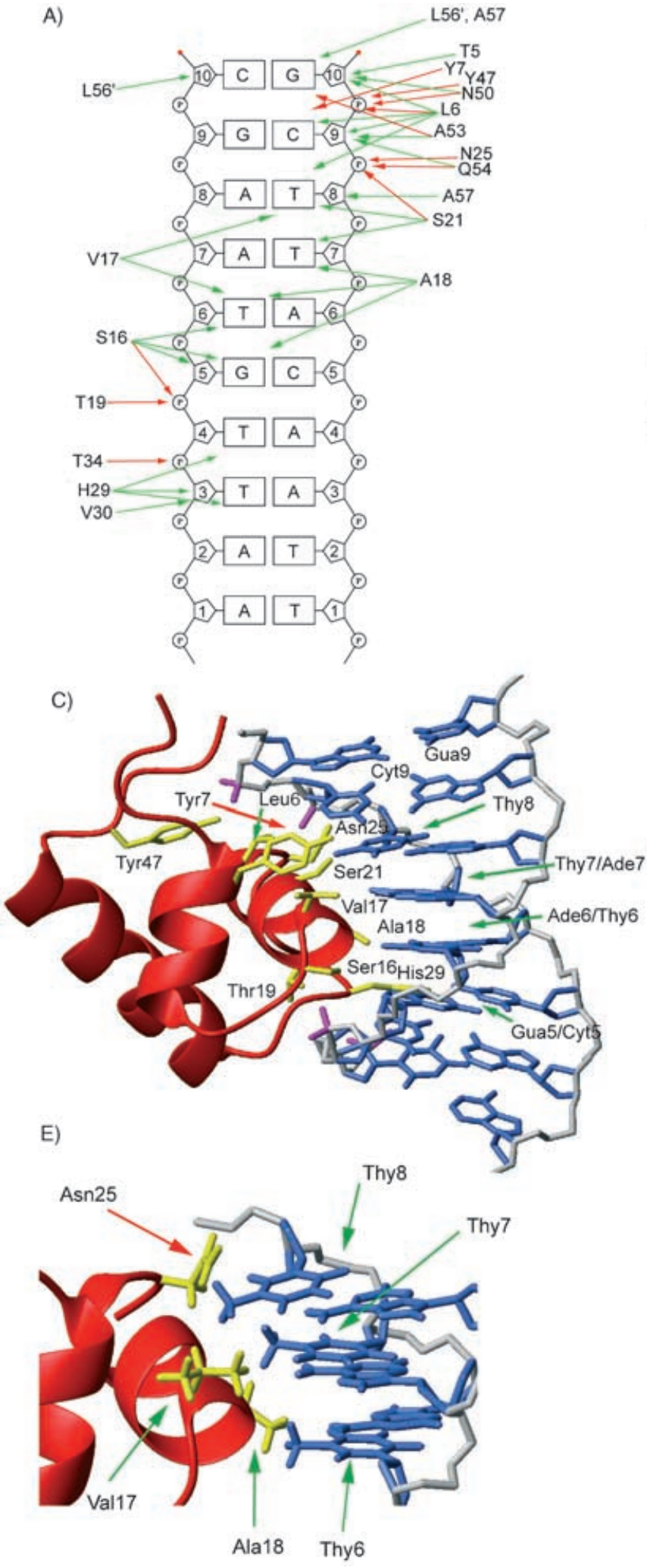

B)
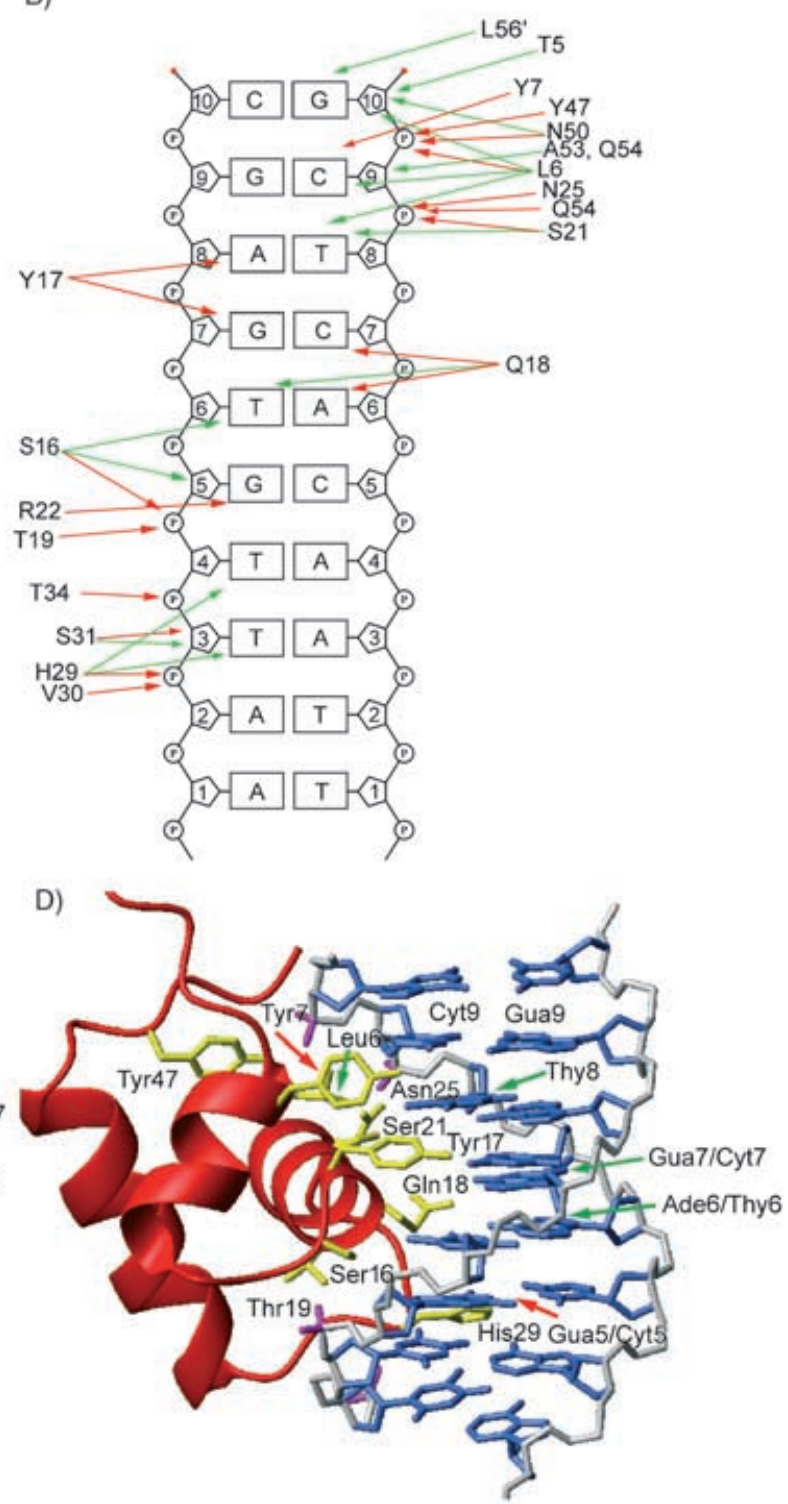

F

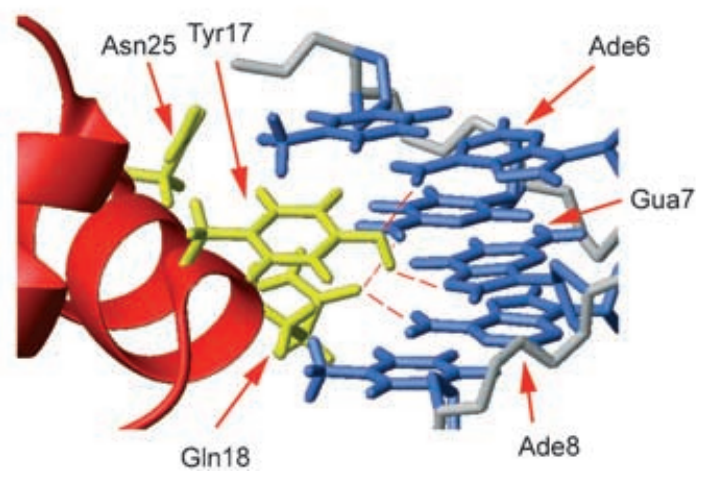

Figure 5. Protein-DNA interactions observed in the Val17Ala18 (left) and in the wild-type lac headpiece DNA complexes (right). A), B) Schematic diagram showing hydrophobic contacts in green and hydrogen bond contacts in red. Only contacts that appear in at least $50 \%$ of the structures in the NMR ensemble are shown. C), D) Details of the structures of the complexes, showing some of the interactions made by residues at the recognition helix with the bases and the backbone in the DNA major groove. Phosphate oxygens of the DNA that accept hydrogen bonds from protein backbone or side chain atoms are depicted in magenta. Red or green arrows are used according to the hydrogen bonding or hydrophobic interactions involved, as described in the diagram above. E), F) Detailed view of the interactions between Ala17 and Val18 side chains and Thy6, Thy7, and Thy 8 methyl groups in the case of the Val17Ala18 headpiece-DNA complex, or the interactions between Tyr17 and Gln18 with Gua7, Ade8, Cyt7, Ade6, and Thy6. Hydrogen atoms were included in the representation of the protein side chains and of the bases of the DNA. Hydrogen bonds are represented by segmented red lines. 
induces opening of the minor groove in order to accommodate the side chains of Leu56 that penetrate the C10pG11 base pair step. ${ }^{[5,11]}$

\section{Discussion}

We have described DNA-binding studies and the solution structure of an altered specificity mutant, the Val17Ala18 lac headpiece, bound to the lac-gal operator, in which base pair 7 of the lac operator has been changed to an AT base pair.

By determining the solution structure of the mutant headpiece in complexation with the lac-gal operator, we provide structural insight into DNA specificity. By gel retardation assays we showed that the dimeric Val17Ala18 lac headpiece recognizes the lac-gal operator better than the wild-type headpiece in vitro, confirming what had previously been proposed for the whole Val17Ala18 lac repressor on the basis of in vivo experiments. ${ }^{[4]}$ Interestingly, we also demonstrated that the Val17Ala18 headpiece mimics the gal repressor by binding and bending the natural gal Oe operator. This suggests that the binding mode of the gal repressor to its natural operator is compatible with the way in which the lac headpiece binds to the lac operator.

\section{Comparison with the structure of the wild-type lac head- piece bound to the left side of the lac operator}

In the wild-type lac headpiece bound to the lac operator, both Tyr17 and Gln18 recognize GC7 through hydrogen bonding contacts. Gua7 accepts a hydrogen bond from Tyr17 $\mathrm{OH}$, while Cyt7 donates a hydrogen bond to Gln18 (Figure 5).

Comparison of the structures of the Val17Ala18 lac headpiece and the wild-type headpiece bound to DNA (Figure 5) shows that both the Val17 and Ala18 pair and the Tyr17 and Gln18 pair contact the base pairs 6, 7, and 8 in the operator, making hydrophobic or hydrogen bonding contacts according to the hydrophobic or polar natures of the side chains and the bases involved.

Analysis of the other protein-DNA contacts in the structure of the wild-type lac headpiece bound to the lac operator shows that all the other residues that would be expected to contact the lac operator do contact the lac-gal operator (Figure 5). Therefore, the orientations of those side chains that make specific contacts to the bases in the major groove of the lac operator, such as Leu6, Tyr7, Ser21, Arg22, and His29, seem to be very similar in both the Val17Ala18 headpiece and the lac headpiece DNA complexes. The same conclusion holds for those side chains that only make contacts to the DNA backbone, such as Thr5, Ser16, Thr19, Asn25, Val30, Ser31, Thr34, or Tyr47 (Figure 5).

\section{The structural basis for the specificity change}

The isolation of mutant repressors that display altered DNAbinding specificity has been described for other HTH transcription regulators. Wharton and Ptashne (1985) demonstrated that, by redesigning the recognition helix of the bacteriophage
434 repressor, it was possible to change its specificity to that of the P22 repressor. ${ }^{[\mathrm{bb}]}$ Similarly, Wharton et al. (1984) showed that the specificity of the 434 repressor could be switched to that of the cro protein by redesigning the recognition helix. ${ }^{[19]}$

Comparison of the protein-DNA contacts observed in the structure of the Val17Ala18 headpiece bound to the lac-gal operator with those observed in the structure of the wild-type lac headpiece bound to the left side of the lac operator shows that, while Val17 and Ala18 make a different network of interactions, the orientation of most side chains that contact the major groove of the DNA is surprisingly conserved (Figure 5). We propose that the contacts made by the side chains of Thr5, Leu6, Ser16, Thr19, and Tyr47 to the DNA backbone are anchoring contacts that help the headpiece to assemble correctly on the lac operator. Once these anchoring points are established, the specificity is then provided by the hydrophobic interactions made by Val17 and Ala18 with the base pairs 6, 7, and 8 on the major groove of the lac-gal operator.

The hydrophobic interaction between Ala18 $\mathrm{H}_{\alpha}$ and $\beta-\mathrm{CH}_{3}$ groups and the methyl group of Thy7 contribute to the stabilization of the protein-DNA complex to a large extent. Similarly, the hydrogen bond contacts from Tyr17 and Gln18 to GC7 must make a large contribution to the stabilization of the wildtype complex (Figure 5). The specificity of the Val17Ala18 headpiece for the lac-gal operator is therefore driven by the complementarity of the protein-DNA interface.

\section{Broad specificity of the Val17 Ala18 headpiece}

Gel retardation experiments confirmed the much higher affinity of the Val17Ala18 headpiece for the lac-gal operator than for the lac-SymL operator under high salt conditions, thus indicating a clear specificity change upon mutation of these two residues.

However, the effects of the salt concentration on the binding affinities of the Val17Ala18 lac headpiece to the lac-gal and to the lac-SymL operators shown in Figure 2 and Table 1 indicate that the Val17Ala18 headpiece displays a lower specificity than the wild-type headpiece under low salt conditions. A detailed inspection of the $K_{\mathrm{d}}$ values reported in Table 1 shows that the Val17Ala18 headpiece binds more tightly than the wild-type headpiece to nonoperator DNA under either low or high salt conditions. This indicates that the mutant headpiece requires higher salt concentrations in order to decrease the contribution of nonspecific binding in the recognition mechanism. It is not clear why the Val17Ala18 headpiece binds more tightly than the wild-type lac headpiece to nonoperator DNA.

It is remarkable that, at low salt concentrations, the Val17Ala18 headpiece binds tightly to the lac-SymL operator and also bends this operator. Similarly, a very weak binding of the wild-type lac headpiece on the lac-gal operator is detected under low salt concentrations and the fraction of the DNA bound is also bent by approximately $33^{\circ}$ (Table 1 ). However, DNA bending was not observed in the NMR structure of the dimeric lac headpiece bound to a nonspecific DNA sequence; this observation was confirmed by our present biochemical data (Table 1). ${ }^{[12]}$ 
The above observations can be related to studies of the interaction of wild-type lac repressor with lac operator variants containing single symmetric substitutions ${ }^{[20 a]}$ and lac operators containing constitutive mutations. ${ }^{[20 \mathrm{~b}]}$ These studies showed that the binding mode to these altered operators can retain some of the thermodynamic characteristics of the binding to the wild-type operator: namely, the polyelectrolyte effect and the heat capacity change of association. It has been proposed that in all cases the protein conformation adapts to the new DNA sequence in such a way as to optimize the interactions at the interface in a particular way for each sequence. ${ }^{[20]}$

\section{Comparison with mutagenesis data}

The orientations of the side chains of Val17 and Ala18 in our structure seem to be compatible with several observations made by Lehming et al. based on experiments using single-residue mutant lac repressors containing Val at position 1 or Ala at position 2 of the recognition helix. ${ }^{[4 c]}$

Lehming et al. also noticed that the complex between the double mutant Val17Ala18 lac repressor and the equivalent lac-gal operator (341 in their nomenclatur ${ }^{[4 \mathrm{c}]}$ ) was much more stable than the complexes between the Val17 or Ala18 singlemutant repressors and the lac-gal operator, suggesting that the combination of residues at positions 1 and 2 of the recognition helix forms a more favorable interface to interact with base pairs 6 and 7 of the lac operator under physiological salt conditions.

The preference for Val and Ala at positions 1 and 2, respectively, was studied by Sartorius et al., who screened several other repressor mutants that would be able to recognize the variant lac operator with AT at position 7, using similar in vivo assays for detecting repression. ${ }^{[4]}$ Mutants containing Ser17Ala18, Ser17Thr18, Pro17Thr18, Ile17Thr18, and His17Val18 were found; this suggests a strong preference for Ala or Thr in position 2, while a larger degree of variability is allowed for position 1 .

\section{Implications for the recognition of the gal operator by the gal repressor}

Probably as a consequence of its broad specificity, the Val17Ala18 headpiece binds, although with lower affinity, to the gal Oe operator (Figure 2 and Table 1). Moreover, it also bends the gal Oe operator upon binding (Figure 3, Table 1).

Figure 1D shows that the left-hand side of the gal Oe operator differs from the lac-gal operators at positions 3 and 9 while the right-hand side differs from the lac-gal operator at position 15. The base pair at position 15 is the symmetric equivalent of position 8 (Figure $1 \mathrm{D}$ ); it is specifically recognized by Val17 and Ala18 in the Val17Ala18 headpiece-DNA complex (Figure 5). It therefore appears reasonable that the Val17Ala18 headpiece should display broad specificity and be able to recognize a GC at that position. In fact, Sartorius et al. detected lower levels of $\beta$-galactosidase repression in the interaction of the Val17Ala18 lac repressor with variant operators containing $C, G$, or $A$ at this position. ${ }^{[4]}$
The sequence alignment of the DNA-binding domains of lac and gal repressors shows that several amino acids that contact the DNA in the lac headpiece-DNA complex are conserved in the gal repressor (Figure $1 \mathrm{~A}){ }^{[8 b]}$ This is the case for Thr4 and lle 5 in the gal, which correspond to Thr 5 and Leu6 in the lac repressor, or galR Ser14, Thr17, Asn23, Ala28, Ser29, and Tyr45, which correspond to lacR Ser16, Thr19, Asn25, Val30, Ser31, and Tyr47. Moreover, the amino acid sequences of the recognition helices of the gal and lac repressors differ only in positions 1, 2, and 8 (Figure 1 A, B). It therefore appears valid to propose that the gal headpiece recognizes the gal Oe operator in a similar way to that in which the Val17Ala18 lac headpiece recognizes the lac-gal operator. The base pairs TA6, AT7, and AT8 on the left-hand side, or TA13, TA14, and CG15 on the right-hand side of the gal Oe operator (Figure 1D), would then participate in specific hydrophobic interactions with Val and Ala at positions 1 and 2 of the gal repressor recognition helix.

The fact that the Val17Ala18 headpiece was able to bend the gal Oe operator suggests that the DNA-bending mechanisms of gal and lac repressors are similar. Although the sequence identity between the lac and the gal repressors in the hinge region is lower than in the recognition helix, Leu56which intercalates in the minor groove of the lac operator-is conserved in the primary sequence of the gal repressor.

\section{Experimental Section}

Expression and purification of the Val1Ala2 lac headpiece: The plasmid pET-HP62(Y17V/Q18A/V52C) was constructed by combination of the plasmid pET-HP62(V52C) with the plasmid pET-HP56 containing the substitutions $\mathrm{Y} 17 \mathrm{~V}$ and $\mathrm{Q} 18 \mathrm{~A}^{[9,21]}$ by use of the Sall and $\mathrm{Sacl}$ restriction sites present in the gene for the lac headpiece. The nucleotide sequence of the plasmid pET-HP62(Y17V/Q18A/ V52C) coding for the Va17Ala18 lac headpiece was confirmed by DNA sequencing. The protein was expressed in E. coli $\mathrm{DH} 9$ by use of a T7 RNA polymerase/promoter system. ${ }^{[2,23]}$ Production and isolation of uniformly ${ }^{15} \mathrm{~N}$ - or ${ }^{13} \mathrm{C} /{ }^{15} \mathrm{~N}$-labeled proteins followed the procedure described for the dimeric wild-type lac headpiece. ${ }^{[9,23]}$ All samples were concentrated with the aid of Amicon concentrators and ultrafiltration membranes from Millipore. The HPLC-purified lac-gal operator (5'-GAATTGTAAGCGCTTACAATTC-3') was purchased from RNA-TEC (Leuven, Belgium); the lac-SymL operator (5'-GAATTGTGAGCGCTCACAATTC-3') was purchased from Carl Roth $\mathrm{GmbH}$ (Karlsruhe, Germany).

DNA-binding and DNA-bending assays: The gel retardation and the DNA-bending assays were performed essentially as described in Spronk et al. (1999). ${ }^{[24]}$ Binding experiments were performed with 140 bp DNA fragments containing the 22 bp lac-gal, lac-SymL, and nonoperator DNA (NOD), and the $23 \mathrm{bp}$ gal Oe operator sites (Figure 1C, D) ligated in the center. These fragments were the same as those used in the bending experiments. All the experiments were performed at $4{ }^{\circ} \mathrm{C}$ in $20 \mu \mathrm{L}$ of reaction buffer containing Tris $(\mathrm{pH} 8.1,10 \mathrm{~mm}), \mathrm{KCl}(50 \mathrm{~mm}$ or $250 \mathrm{~mm})$, EDTA $(1 \mathrm{~mm})$, glycerol $(5 \% \mathrm{v} / \mathrm{v})$, and BSA $\left(0.1 \mathrm{mg} \mathrm{mL}^{-1}\right)$.

NMR spectroscopy: The NMR samples contained dimeric ${ }^{15} \mathrm{~N}$ - or ${ }^{15} \mathrm{~N} /{ }^{13} \mathrm{C}$ double-labeled Val1Ala2 lac headpiece $(1 \mathrm{~mm})$ and unlabeled lac-gal operator $(1 \mathrm{~mm})$ in $\mathrm{KPi}$ buffer $(\mathrm{pH} 6.0,10 \mathrm{~mm}), \mathrm{KCl}$ $(20 \mathrm{~mm}),\left[\mathrm{D}_{8}\right]$ glycerol $(5 \%), \mathrm{D}_{2} \mathrm{O}(5 \%)$, and $\mathrm{NaN}_{3}(0.1 \%)$. All the NMR spectra were acquired at $315 \mathrm{~K}$ on Bruker AVANCE spectrome- 
ters. Backbone resonance assignments were based on previous assignments available for the wild-type lac headpiece bound to the lac operator and on the analysis of the sequential connectivities observed in 3D NOESY- $\left({ }^{1} \mathrm{H},{ }_{1}^{15} \mathrm{~N}\right)-\mathrm{HSQC}$ spectra. Confirmation of these assignments was achieved by analysis of $3 \mathrm{D} \mathrm{CBCA(CO)NH}$ and $3 \mathrm{D}$ HNCO experiments recorded on spectrometers operating at 600 and $750 \mathrm{MHz}$. Side chain assignments were obtained from a 2D $\left({ }^{1} \mathrm{H}^{13} \mathrm{C}\right) \mathrm{HSQC}$ modified for aromatic resonances, 3D HBHA(CO)NH, 3D $(\mathrm{H}) \mathrm{CC}(\mathrm{CO}) \mathrm{NH}, 3 \mathrm{D} \mathrm{HC}(\mathrm{C}) \mathrm{H}-\mathrm{TOCSY}$, and 3D $(\mathrm{H}) \mathrm{CCH}-$ TOCSY experiments acquired at $700 \mathrm{MHz}$ or on a $600 \mathrm{MHz}$ spectrometer fitted with a cryoprobe. Intramolecular NOEs for the protein were identified from 2D NOE, 3D NOESY $-\left({ }^{1} \mathrm{H}^{15} \mathrm{~N}\right)-\mathrm{HSQC}$, and 3D NOESY- $\left({ }^{1} \mathrm{H}^{13} \mathrm{C}\right)$-HSQC experiments acquired at 750 or $900 \mathrm{MHz}$ with a mixing time of $100 \mathrm{~ms}$. The assignment of the DNA resonances and the collection of intramolecular DNA NOEs and intermolecular protein-DNA NOEs were performed in a $2 \mathrm{D}$ NOE spectrum acquired at $900 \mathrm{MHz}$, and ${ }^{13} \mathrm{C} /{ }^{15} \mathrm{~N}$ double-filtered 2D NOE experiments acquired in water and in $\mathrm{D}_{2} \mathrm{O}$ at 700 and $900 \mathrm{MHz}$, with a mixing time of $100 \mathrm{~ms}$. One protein-DNA hydrogen bond involving the backbone HN of Leu6 and a phosphate group of the DNA could be identified by the observation of a cross hydrogen bond scalar coupling ${ }^{3} J_{\mathrm{NP}}$ in a ${ }^{31} \mathrm{P}$-edited constant time $\left({ }^{1} \mathrm{H},{ }^{15} \mathrm{~N}\right) \mathrm{HSQC}$ experiment performed with and without ${ }^{31} \mathrm{P}$ decoupling during the constant time delay of $60 \mathrm{~ms} .{ }^{[25]}$ The observation of the $\mathrm{OH}$ signal of Tyr47 indicated that this atom was protected from exchange with the solvent, suggesting that it was also participating in a hydrogen bond. Residual ${ }^{1} D_{\mathrm{NH}}$ and ${ }^{1} D_{\mathrm{CH}}$ dipolar couplings were determined from the differences in measured ${ }^{1} J_{\mathrm{NH}}$ and ${ }^{1} J_{\mathrm{CH}}$ values at two different magnetic fields: $21.14 \mathrm{~T}\left({ }^{1} \mathrm{H}\right.$ frequency $\left.900.21 \mathrm{MHz}\right)$ and $11.75 \mathrm{~T}$ (' $\mathrm{H}$ frequency $500.28 \mathrm{MHz}$ ) due to the intrinsic anisotropic magnetic susceptibility of DNA. The values for the ${ }^{1} J_{N H}$ and ${ }^{1} J_{C H}$ couplings were extracted from $J$-modulated $2 \mathrm{D}$ HSQC experiments as described by Tjandra et al. ${ }^{[26]}$ The spectra were acquired with 256 complex points in the indirect dimension, and a constant time (CT) period of $28 \mathrm{~ms}$ for the $\mathrm{CT}-{ }^{1} \mathrm{H}^{13} \mathrm{C}-\mathrm{HSQC}$ experiments. All spectra were processed with NMRPipe and analyzed by using NMRView.5.0.4. ${ }^{[27]}$

NMR restraints: Intra- and intermolecular NOEs were analyzed and converted into distance restraints by use of NMRView. ${ }^{[27 b]}$ Chemical shifts of ${ }^{15} \mathrm{~N}, \mathrm{H}_{\alpha^{\prime}}{ }^{13} \mathrm{C}_{\alpha,}{ }^{13} \mathrm{C}_{\beta}$, and ${ }^{13} \mathrm{C}^{\prime}$ resonances were used as input for TALOS to predict $\varphi$ and $\psi$ dihedral angles, which were subsequently used as restraints in the structure calculations. ${ }^{[28]}$ Two protein-DNA hydrogen bonds connecting Leu6 $\mathrm{HN}$ and Tyr47 $\mathrm{OH}$ with the phosphate group of Cyt9 were also used as intermolecular restraints. The assignment of these hydrogen bonds is based on the observations described previously, and also on the analysis of preliminary structures in which both Leu6 $\mathrm{HN}$ and Tyr47 $\mathrm{OH}$ hydrogen bond the phosphate group of Cyt9; these contacts have also been observed in the wild-type complex. They were introduced as ambiguous restraints to either of the two oxygen atoms of the phosphate group of $\mathrm{Cyt} 9$, with upper bounds of $2 \AA(\mathrm{H}-\mathrm{OP})$ or $3 \AA(\mathrm{N}$ $\mathrm{OP}$, or O-OP). NOEs that could have a contribution from proteinprotein intermonomer contacts were assigned on the basis of similarity with the previously described wild-type lac-headpiece-lac operator complexes. ${ }^{[4,11]}$ They were treated as ambiguous intra- and intermonomer restraints. RDCs were included as harmonic restraints. ${ }^{[29]}$ The value of the RDC force constant $\left(100 \mathrm{kcal} \mathrm{mol}^{-1} \mathrm{~Hz}^{-2}\right)$ was chosen such that the difference between the back-calculated and the experimentally determined values was roughly in agreement with the experimental error. The axial and rhombic components of the molecular alignment tensor $D a$ and $R$, respectively, were initially determined by the histogram method as implemented in PALES2.0, ${ }^{[30 a, c]}$ and further refined by a grid search procedure. ${ }^{[30 \mathrm{~b}]}$ By using this approach, the optimal values were found to be $D a=1.1 \mathrm{~Hz}$ and $R=0.65$.

Structure calculation of the dimer: The calculation of the symmetric Val17Ala18 lac headpiece dimer was performed with ARIA1.3 protocols by use of $\mathrm{CNS}^{\left[{ }^{[31 a-b]}\right.}$ with the parallhdg5.3 force field and the PROLSQ parameter set. ${ }^{[31 c-d]}$ The structure was initially calculated on the basis of distance and dihedral angle restraints as described previously for the wild-type complex. ${ }^{[49]}$ The 20 structures with lowest total energy were further refined with the $D_{\mathrm{NH}}$ restraints in a second simulated annealing protocol in torsion angle space, also including C2 and noncrystallographic (NCS) symmetry restraints. ${ }^{[32]} 200$ structures were calculated, and 50 of them were refined in explicit water. An ensemble of 12 structures was selected for docking on the DNA on the basis of low total energy and suitable orientation of the two monomers, in agreement with the previously determined wild-type complex.

Structure calculation of the protein-DNA complex: Docking on the lac-gal operator was performed with HADDOCK1.3 with use of $\mathrm{CNS}^{[33,31 \mathrm{~b}]}$ the parallhdg5.3 force field, and the OPLS nonbonded parameter set with full electrostatic and van der Waals potentials with use of an $8.5 \AA$ cutoff and a dielectric constant of 10 for the vacuum part. ${ }^{[31,, 34]}$ The starting B-DNA structure was built by use of Namot2 (www.t10.lanl.gov/namot/). Watson-Crick base pairing and the planarity of purine and pyrimidine rings were ensured by a set of artificial restraints. ${ }^{[49]}$ Backbone dihedral angle restraints for BDNA were used in order to maintain the conformations of the first seven and the last seven base pairs of the DNA (bps 1-7 and 1622). Symmetry restraints were used in order to maintain the $C 2$ symmetry of the complex during the whole docking procedure. The docking strategy consisted of a first step of rigid body minimization with DNA and protein $50 \AA$ away from each other. The actual docking was performed during the simulated annealing stage in torsion angle space, with the protein and the eight central DNA base pairs treated as fully flexible $(5000$ steps at $2000 \mathrm{~K}, 8000$ steps from 2000 to $500 \mathrm{~K}$, and a final 8000 steps from $2000 \mathrm{~K}$ to $50 \mathrm{~K}$, with the remainder of the DNA treated as flexible as well). In total, 200 structures were generated, and the 50 lowest-energy structures were selected for refinement in explicit water including the $D_{\mathrm{NH}}$ restraints. The 20 lowest-energy structures were selected for analysis. Protein-DNA interactions were analyzed with DIM$\mathrm{PLOT}^{[35]}$ the DNA helical parameters and the bend angle were analyzed with CURVES, ${ }^{[16]}$ and the RDC quality factor (Q-factor ${ }^{[18]}$ ) was evaluated with PALES. ${ }^{[30 c]}$ The figures depicting the structure were generated with MOLMOL. ${ }^{[36]}$ The atomic coordinates of the final structures have been deposited in the Protein Data Bank (accession code 2BJC).

\section{Acknowledgements}

This work was financially supported by the Chemical Division of the Netherlands Organization for Scientific Research (NWO-CW). R.K.S. was the recipient of a Ph.D. fellowship from the National Council for Scientific and Technological Development (CNPq, Brazil). We thank E. Halff for her assistance in preparing the pBend2-lac-gal vector, R. Wechselberger and E. Tischenko for technical assistance in acquiring some of the NMR spectra, A. George for technical assistance with protein expression and purification, and Dr. D. L. Bryce for providing scripts to predict the theoretical values of DNA magnetic susceptibility tensor anisotropy and rhombicity. 
Keywords: DNA recognition - NMR spectroscopy · proteinDNA recognition $\cdot$ specificity $\cdot$ transcription

[1] a) C. G. Kalodimos, R. Boelens, R. Kaptein, Chem. Rev. 2004, 104, 3567 3586; b) H. C. Pace, M. A. Kercher, P. Lu, P. Merkiewicz, J. H. Miller, G. Chang, M. Lewis, Trends Biochem. Sci. 1997, 22, 334-339; c) B. MüllerHill, Curr. Opin. Microbiol. 1998, 1, 145-151; d) C. E. Bell, M. Lewis, Curr. Opin. Struct. Biol. 2001, 11, 19-25.

[2] C. O. Pabo, R. T. Sauer, Annu. Rev. Biochem. 1984, 53, 293-321.

[3] a) R. H. Ebright, P. Cossart, B. Gicquel-Sanzey, J. Beckwith, Nature 1984 311, $232-235$; b) R. P. Wharton, M. Ptashne, Nature 1985, 316, $601-605$ c) R. G. Brennan, B. W. Matthews, J. Biol. Chem. 1989, 264, 1903-1906 d) S. C. Harrison, A. K. Aggarwal, Annu. Rev. Biochem. 1990, 59, 933-969.

[4] a) R. H. Ebright, Proc. Natl. Acad. Sci. USA 1986, 83, 303-307; b) R. Boelens, R. M. J. N. Lamerichs, J. A. C. Rullmann, J. H. van Boom, R. Kaptein Protein Sequences Data Anal. 1988, 1, 487-498; c) N. Lehming, J. Sartorius, M. Niemoller, G. Genenger, B. von Wilcken-Bergmann, B. Müller-Hill, $E M B O$ J. 1987, 6, 3145-3153; d) N. Lehming, J. Sartorius, S. Oehler, B. von Wilcken-Bergmann, B. Müller-Hill, Proc. Natl. Acad. Sci. USA 1988, 85, 7947-7951; e) J. Sartorius, N. Lehming, B. Kisters, B. von WilckenBergmann, B. Müller-Hill, EMBO J. 1989, 8, 1265-1270; f) L. G. Kleina, J. Miller, J. Mol. Biol. 1990, 212, 295-318; g) C. G. Kalodimos, A. M. J. J. Bonvin, R. K. Salinas, R. Wechselberger, R. Boelens, R. Kaptein, EMBO J. 2002, 21, 2866-2876

[5] a) C. A. E. M. Spronk, M. Slijper, J. H. van Boom, R. Kaptein, R. Boelens, Nat. Struct. Biol. 1996, 3, 916-919; b) M. Lewis, G. Chang, N. C. Horton M. A. Kercher, H. C. Pace, M. A. Schumacher, R. Brennan, P. Lu, Science 1996, 271, 1247-1254.

[6] N. Lehming, J. Sartorius, B. Kisters-Woike, B. von Wilcken-Bergmann, B. Müller-Hill, EMBO J. 1990, 9, 615-621.

[7] A. Simons, D. Tils, B. von Wilcken-Bergmann, B. Müller-Hill, Proc. Natl. Acad. Sci. USA 1984, 81, 1624-1628.

[8] a) W. Gilbert, A. Maxam, Proc. Natl. Acad. Sci. USA 1973, 70, $3581-3584$ b) B. von Wilcken-Bergmann, B. Muller-Hill, Proc. Natl. Acad. Sci. USA 1982, 79, 2427-2431; c) A. Majumdar, S. Adhya, J. Biol. Chem. 1987, $262,13258-13262$.

[9] C. G. Kalodimos, G. E. Folkers, R. Boelens, R. Kaptein, Proc. Natl. Acad. Sci. USA 2001, 98, 6039-6044.

[10] H. Wu, D. M. Crothers, Nature 1984, 308, 509-513.

[11] C. A. E. M. Spronk, A. M. J. J. Bonvin, P. Radha, G. Melacini, R. Boelens, R. Kaptein, Structure 1999, 7, $1483-1492$.

[12] C. G. Kalodimos, N. Biris, A. M. J. J. Bonvin, M. M. Levandoski, M. Guennuegues, R. Boelens, R. Kaptein, Science 2004, 305, 386- 389.

[13] C. Zwieb, J. Kim, S. Adhya, Genes Dev. 1989, 3, 606-611.

[14] M. Slijper, A. M. J. J. Bonvin, R. Boelens, R. Kaptein, J. Mol. Biol. 1996, $259,761-773$

[15] V. P. Chuprina, J. A. C. Rullmann, R. M. J. N. Lamerichs, J. H. van Boom, R. Boelens, R. Kaptein, J. Mol. Biol. 1993, 234, 446-462.

[16] R. Lavery, H. Sklenar, J. Biomol. Struct. Dyn. 1988, 6, 63-91.
[17] a) N. Tjandra, S. Tate, A. Ono, M. Kainosho, A. Bax, J. Am. Chem. Soc 2000, 122, 6190-6200; b) H. C. Kung, K. Y. Wang, I. Goljer, P. Bolton, J. Magn. Reson. Ser. B 1995, 109, 323-325.

[18] G. Cornilescu, J. L. Marquardt, M. Ottiger, A. Bax, J. Am. Chem. Soc. $1998,120,6836-6837$.

[19] R. P. Wharton, E. L. Brown, M. Ptashne, Cell 1984, 38, 361-369.

[20] a) D. E. Frank, R. M. Saecker, J. P. Bond, M. W. Capp, O. V. Tsodikov, S. E. Melcher, M. M. Levandoski, M. T. Record, J. Mol. Biol. 1997, 267, 11861206; b) M. C. Mossing, M. T. Record, J. Mol. Biol. 1985, 186, 295-305.

[21] M. Slijper, Ph.D. Thesis, Utrecht University (The Netherlands), 1996.

[22] a) D. L. Hare, J. R. Sadler, Gene 1978, 3, 269-278; b) S. Tabor, C. C. Richardson, Proc. Natl. Acad. Sci. USA 1985, 82, 1074-1078.

[23] M. Slijper, R. Boelens, A. L. Davis, R. N. H. Konings, G. A. van der Marel, J. H. van Boom, R. Kaptein, Biochemistry 1997, 36,249-254.

[24] C. A. E. M. Spronk, G. E. Folkers, A. G. W. Noordman, R. Wechselberger, N. van den Brink, R. Boelens, R. Kaptein, EMBO J. 1999, 18, 6472-6480.

[25] M. Mishima, M. Hatanaka, S. Yokoyama, T. Ikegami, M. Walchli, Y. Ito, M. Shirakawa, J. Am. Chem. Soc. 2000, 122, 5883-5884.

[26] a) N. Tjandra, S. Grzesiek, A. Bax, J. Am. Chem. Soc. 1996, 118, 62646272 ; b) N. Tjandra, A. Bax, J. Magn. Reson. 1997, 124, 512-515.

[27] a) F. Delaglio, S. Grzesiek, G. W. Vuister, G. Zhu, J. Pfeifer, A. Bax, J. Biomol. NMR 1995, 6, 277-293; b) B. A. Johnson, R. A. Blevins, J. Biomol. NMR 1994, 4, 603-614.

[28] G. Cornilescu, F. Delaglio, A. Bax, J. Biomol. NMR 1999, 13, 289-302.

[29] N. Tjandra, J. G. Omichinski, A. M. Gronenborn, G. M. Clore, A. Bax, Nat. Struct. Biol. 1997, 4, 732-738.

[30] a) G. M. Clore, A. M. Gronenborn, A. Bax, J. Magn. Reson. 1998, 133, 216-221; b) G. M. Clore, A. M. Gronenborn, N. Tjandra, J. Magn. Reson. 1998, 131, 159-162; c) M. Zweckstetter, A. Bax, J. Am. Chem. Soc. 2000, $122,3791-3792$.

[31] a) J. P. Linge, M. Habeck, W. Rieping, M. Nilges, Bioinformatics 2003, 19 315-316; b) A. T. Brunger, P. D. Adams, M. G. Clore, W. L. DeLano, P. Gros, R. W. Grosse-Kunstleve, J. Jiang, J. Kuszewski, M. Nilges, N. S. Pannu, R. J. Read, L. M. Rice, T. Simonson, G. L. Warren, Acta Crystallogr. Sect. D Biol. Crystallogr. 1998, 54, 905-921; c) J. P. Linge, M. A. Williams, C. A. E. M. Spronk, A. M. J. J. Bonvin, M. Nilges, Proteins 2003, 50, $496-$ 506; d) R. A. Engh, R. Huber, Acta Crystallogr. Sect. A Found. Crystallogr. $1991,47,392-400$

[32] M. Nilges, Proteins 1993, 17, 297-309.

[33] C. Dominguez, R. Boelens, A. M. J. J. Bonvin, J. Am. Chem. Soc. 2003, $125,1731-1737$.

[34] W. L. Jorgensen, J. Tirado-Rives, J. Am. Chem. Soc. 1988, 110, 16571671.

[35] A. C. Wallace, R. A. Laskowski, J. M. Thornton, Prot. Eng. 1995, 8, $127-$ 134.

[36] R. Koradi, M. Billeter, K. Wüthrich, J. Mol. Graphics 1996, 14, 51-55.

[37] D. L. Bryce, J. Boisbouvier, A. Bax, J. Am. Chem. Soc. 2004, 126, $10820-$ 10821.

Received: February 4, 2005

Published online on August 11, 2005 